\title{
Perspective
}

\section{On the successes and opportunities for discovery of metal oxide photoanodes for solar fuels generators}

Lan Zhou, Aniketa Shinde, Dan Guevarra, Joel A. Haber, Kristin A. Persson, Jeffrey B. Neaton, and John M. Gregoire

ACS Energy Lett., Just Accepted Manuscript • Publication Date (Web): 31 Mar 2020

Downloaded from pubs.acs.org on March 31, 2020

\section{Just Accepted}

"Just Accepted" manuscripts have been peer-reviewed and accepted for publication. They are posted online prior to technical editing, formatting for publication and author proofing. The American Chemical Society provides "Just Accepted" as a service to the research community to expedite the dissemination of scientific material as soon as possible after acceptance. "Just Accepted" manuscripts appear in full in PDF format accompanied by an HTML abstract. "Just Accepted" manuscripts have been fully peer reviewed, but should not be considered the official version of record. They are citable by the Digital Object Identifier (DOI@). "Just Accepted" is an optional service offered to authors. Therefore, the "Just Accepted" Web site may not include all articles that will be published in the journal. After a manuscript is technically edited and formatted, it will be removed from the "Just Accepted" Web site and published as an ASAP article. Note that technical editing may introduce minor changes to the manuscript text and/or graphics which could affect content, and all legal disclaimers and ethical guidelines that apply to the journal pertain. ACS cannot be held responsible for errors or consequences arising from the use of information contained in these "Just Accepted" manuscripts. 


\title{
On the successes and opportunities for discovery of
}

\section{metal oxide photoanodes for solar fuels generators}

\author{
Lan Zhou, ${ }^{a}$ Aniketa Shinde, ${ }^{a}$ Dan Guevarra, ${ }^{a}$ Joel A. Haber, ${ }^{a}$ Kristin A. Persson, ${ }^{b, c, d}$ Jeffrey B. \\ Neaton, b,e,f John M. Gregoire ${ }^{a, g, *}$ *
}

a Joint Center for Artificial Photosynthesis, California Institute of Technology; Pasadena, California 91125, United States; ${ }^{b}$ Joint Center for Artificial Photosynthesis, Lawrence Berkeley National Laboratory, Berkeley, CA 94720, United States; ${ }^{\mathrm{c}}$ Department of Materials Science and Engineering, University of California, Berkeley, CA 94720, United States; ${ }^{\mathrm{d}}$ Environmental Energy Technologies Division, Lawrence Berkeley National Laboratory; ${ }^{\mathrm{e}}$ Department of Physics, University of California, Berkeley, Berkeley, CA 94720, United States; ${ }^{\mathrm{f}}$ Molecular Foundry, Lawrence Berkeley National Laboratory, Berkeley, CA 94720, United States; Kavli Energy NanoSciences Institute, University of California, Berkeley, Berkeley, CA 94720, United States. ${ }^{\mathrm{g}}$ Division of Engineering and Applied Science, California Institute of Technology, Pasadena, CA 91125, United States

\section{Corresponding Author \\ *gregoire@caltech.edu}

\begin{abstract}
The importance of metal oxide photoanodes in solar fuels technology has garnered concerted efforts in photoanode discovery in recent decades, which complement parallel efforts in
\end{abstract}


development of analytical techniques and optimization strategies using standard photoanodes such as $\mathrm{TiO}_{2}, \mathrm{Fe}_{2} \mathrm{O}_{3}$ and $\mathrm{BiVO}_{4}$. Theoretical guidance of high throughput experiments has been particularly effective in dramatically increasing the portfolio of metal oxide photoanodes, motivating a new era of photoanode development where the characterization and optimization techniques developed on traditional materials are applied to nascent photoanodes that exhibit visible light photoresponse. The compendium of metal oxide photoanodes presented in the present work can also serve as the basis for further technique development, with a primary goal to establish workflows for discovery of materials that perform better against the critical criteria of operational stability, visible light photoresponse, and photovoltage suitable for tandem absorber architectures.

\section{TOC GRAPHICS}

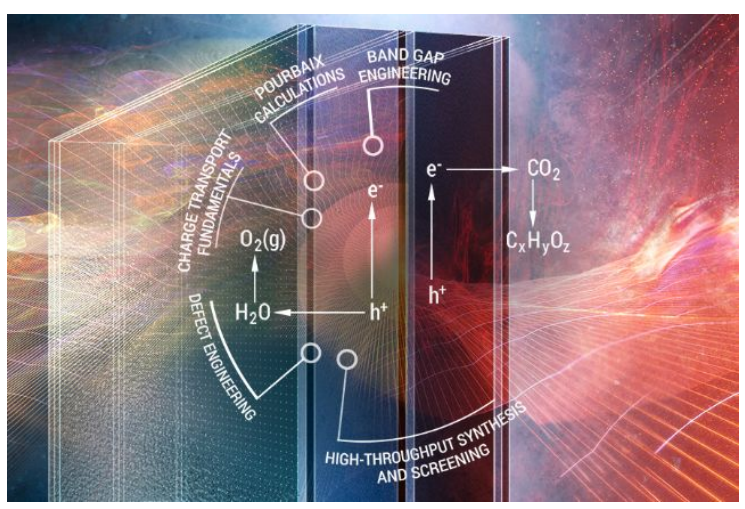

The generation of chemicals and fuels from $\mathrm{CO}_{2}, \mathrm{~N}_{2}$, and $\mathrm{H}_{2} \mathrm{O}$ in photoelectrochemical reactors would enable sustainable energy infrastructure with decreased reliance on photovoltaic and battery technologies that pose challenges for energy storage and transport. ${ }^{1}$ The source chemical $\mathrm{CO}_{2}$ is used for generation of $\mathrm{C}$-containing chemicals such as $\mathrm{CO}$ and formate as well as higher-order fuels such as liquid hydrocarbons and alcohols that could displace fossil-based liquid fuels while leveraging existing infrastructure. ${ }^{2}$ The source chemical $\mathrm{N}_{2}$ is used for generation of $\mathrm{NH}_{3}$ and less 
often $\mathrm{N}_{2} \mathrm{H}_{4}$, which may serve as fuels but can serve a more pressing need of sustainable fertilizer production. ${ }^{3}$ These families of reactions involve electrochemical reduction of the source chemicals, generally referred to as $\mathrm{CO}_{2}$ reduction reactions $\left(\mathrm{CO}_{2} \mathrm{RR}\right)$ and $\mathrm{N}_{2}$ reduction reactions $\left(\mathrm{N}_{2} \mathrm{RR}\right)$, respectively. These reduction reactions require protons and electrons, and liberation of those reactants from $\mathrm{H}_{2} \mathrm{O}$ via the $\mathrm{O}_{2}$ evolution reaction (OER) has been adopted as the primary strategy for establishing broadly-deployable solar fuels technologies. The $\mathrm{H}_{2}$ evolution reaction (HER) can also be coupled to the OER without $\mathrm{CO}_{2}$ or $\mathrm{N}_{2}$ reactants, making solar photoelectrocatalysis of the OER a cross-cutting technology for generation of $\mathrm{H}_{2}, \mathrm{C}$-containing, and N-containing fuels. Metal oxide photoanodes can also be used for anodic reactions other than the OER, resulting in synthesis of other chemicals, ${ }^{4}$ although the present work considers solar fuels photoanodes to be photoelectrocatalysts for the OER in aqueous electrolyte.

While photoelectrocatalysis of the HER and OER can occur with a single wide-gap semiconductor, as demonstrated in the seminal water splitting work utilizing $\mathrm{TiO}_{2},{ }^{5}$ the broad consensus, supported by multi-physics device modelling, ${ }^{6}$ is that efficient utilization of the solar spectrum requires tandem light absorbers with band gap energies in the visible spectrum. While a number of device architectures utilizing a pair of visible-gap semiconductors have been proposed, a grand challenge of the solar fuels community has been the identification of a suitable solar fuels photoanode, i.e. a semiconductor that can utilize visible light to effect photoelectrocatalysis of the OER and circumvent a broad range of deactivation processes such as corrosion.

Photovoltaic-grade semiconductors, most notably III-V semiconductors, have enabled a variety of high efficiency solar water splitting demonstrations. ${ }^{7}$ While protective coating ${ }^{8}$ of photovoltaic semiconductors has been effective in increasing operational stability from minutes to over 100 hours, ${ }^{7}$ semiconductors that don't self-passivate under operational conditions will always be 
susceptible to device failure upon damage in the protective coating(s). This type of single point failure is difficult to circumvent in systems engineering and can render the technology untenable for deployment. Intrinsic stability of the semiconductors is the most reliable way to achieve a durable solar fuels generator, which has motivated the persistent and continuing effort to understand metal oxide semiconductors and identify those that can serve as solar fuels photoanodes. No fundamental limit on the efficiency of metal oxide photoanodes has been established beyond those dictated by the thermodynamics of solar energy conversion, and $\mathrm{BiVO}_{4}-$ based devices with ca. 5\% efficiency are approaching these limits given its $2.4 \mathrm{eV}$ band gap. ${ }^{9}$ Photoanode band gap energies no larger than $2 \mathrm{eV}$ are required to realize the $15 \%-20 \%$ solar to fuel conversion efficiencies, the target range per technoeconomic and device models, ${ }^{6,10}$ requiring the community to pursue a combination of low band gap energy and high radiative efficiency that has yet to be approached by metal oxide photoanodes.

To establish the outlook for this grand challenge, we first summarize the progress to date. Recent reviews have highlighted a broad portfolio of materials, techniques and devices. Abdi and Berglund ${ }^{11}$ recently reviewed metal oxide photoanodes with focus on the optimization of $\mathrm{BiVO}_{4}$ and its implementation into water splitting devices, along with summary of several other $\mathrm{V}, \mathrm{W}$ and Fe-based oxide photoanodes, covering in total 9 metal oxide photoanode phases. Chu et al. ${ }^{12}$ focused more on classes of materials and the integration of photoelectrodes into devices. He et al. ${ }^{13}$ compiled a more detailed survey of metal oxide photoanodes with 33 phases discussed and critical analysis of several topics including the electronic character of the conduction and valence bands and other electronic structure considerations. Our literature survey, summarized in Fig. 1 and detailed in the SI, identified 109 OER metal oxide photoanode phases, including recent discoveries from our labs. Of these, we find 70 visible light-active phases, corresponding to 
photoanodes demonstrated to be photoactive with only sub-3 eV illumination. ${ }^{\S}$ The breadth of elements utilized in photoanode studies is rapidly expanding, and the number of visible light-active metal oxide photoanodes has increased 5 -fold in the past 20 years, an acceleration in discovery driven by the concerted efforts in the community, including our high throughput discovery program in the Joint Center for Artificial Photosynthesis (JCAP). During these 20 years, there has also been substantial effort to develop and deploy the present champion visible-gap metal oxide photoanode, $\mathrm{BiVO}_{4}{ }^{11,} 14$ These parallel community efforts in discovery of new metal oxide photoanodes and in optimization and understanding of $\mathrm{BiVO}_{4}$, both of which have been remarkably successful, provide the framework for the future of the field.

$\mathrm{BiVO}_{4}$ is an exemplar of a complex metal-oxide, visible-light photoanode whose study has established the basis for accelerating development of metal oxide photoanodes. ${ }^{15}$ In addition to optimization schemes such as defect engineering for charge transport and selective carrier extraction, ${ }^{16}$ research on $\mathrm{BiVO}_{4}$ has resulted in development of a broad range of materials and device-level solar fuels characterization techniques. ${ }^{17}$ A recent review of strategies for enhancing the photocurrent, photovoltage, and stability of photoelectrodes highlights the breadth and effectiveness of feedback between synthesis and characterization of device-relevant parameters to optimize materials, as illustrated in Fig. $2 .{ }^{17}$ The breadth of these materials development strategies far exceeds the breadth of candidate photoanodes for which they have been deployed. The general bias in scientific research towards continued investigation of well-researched materials is both broadly known and recently evaluated as a limitation on creativity and discovery, ${ }^{18}$ motivating our effort in the present work to establish the set of known visible light-active photoanodes and discuss opportunities for improving performance both within this set of materials and beyond, via new materials discovery strategies. 
Of the 70 visible light-active metal oxide photoanodes, historically $\mathrm{Fe}_{2} \mathrm{O}_{3}$ and more recently $\mathrm{BiVO}_{4}$ are arguably the only materials for which the community has deeply invested in detailed understanding and optimization, motivating further study on the other 68 phases, or some principled selection of a subset thereof. Some notable efforts in this area include detailed experimental investigation of $\alpha-\mathrm{SnWO}_{4},{ }^{19} \mathrm{Fe}_{2} \mathrm{WO}_{6},{ }^{20}$ copper vanadates such as $\beta-\mathrm{Cu}_{2} \mathrm{~V}_{2} \mathrm{O}_{7}$ and $\gamma$ $\mathrm{Cu}_{3} \mathrm{~V}_{2} \mathrm{O}_{8},{ }^{21}$ and computational investigation of $\beta-\mathrm{Cu}_{2} \mathrm{~V}_{2} \mathrm{O}_{7}{ }^{22}$ to elucidate performance-limiting properties. Of these, only the copper vanadates exhibit a photon energy onset of photoactivity near $2 \mathrm{eV}$, the desired upper-limit described above. Fig. 3 summarizes the photon energy onset for 49 metal oxide photoanodes that exhibit external quantum efficiency (EQE) in excess of $0.01 \%$ in our experiments. Two notable phases with photoactivity at $2.1 \mathrm{eV}$ (but insufficient to meet the EQE threshold) are $\mathrm{VCrO}_{4}$-orth ${ }^{23}$ and $\mathrm{V}_{2} \mathrm{CoO}_{6}$-tri. ${ }^{24}$ The 4 phases that exceed the threshold at $2.1 \mathrm{eV}$ are $\mathrm{FeBiO}_{3}$, discovered by Chen et al., ${ }^{25}$ as well as $\mathrm{FeWO}_{4},{ }^{26} \gamma-\mathrm{V}_{2} \mathrm{Cu}_{3} \mathrm{O}_{8},{ }^{27}$ and $\mathrm{Y}_{3} \mathrm{Fe}_{5} \mathrm{O}_{12},{ }^{24}$ highlighting the challenge of identifying metal oxide photoanodes with broad spectral response. A chopped illumination voltage sweep is shown for each of these 4 phases, demonstrating that the photocurrent decreases quickly with decreasing bias for most phases. Anecdotal examples demonstrating improvement in operational photovoltage, i.e. beyond that exhibited in this figure, include the observation of a turn on potential (lowest potential with observed photocurrent) near $0.6 \mathrm{~V}$ vs $\mathrm{RHE}$ for $\mathrm{FeBiO}_{3}{ }^{25}$ and near $0.4 \mathrm{~V}$ vs $\mathrm{RHE}$ for Bi-alloyed $\mathrm{FeWO}_{4}{ }^{26}$ More detailed understanding of the semiconductor-liquid junctions and band energy alignment are needed to elucidate the limiting photovoltage and associated efficiency of each photoanode, as discussed further below.

With regards to the opportunity space for further discovery, it is interesting to consider the fraction of the metal oxide search space that has been explored. The 70 visible light photoanodes 
utilize 25 cation elements from the periodic table. Considering only ternary metal oxides, which account for all but 6 of these phases, only 34 of the 300 pairwise combinations of these cations have been reported. While some may have been explored without discovery, most remain uncharted territory. High Throughput (HiTp) experimental screening of OER photoanodes, pioneered by Parkinson ${ }^{28}$ and McFarland ${ }^{29}$ and advanced by others, ${ }^{30}$ accelerates exploration of this broad class of photoanode candidates, although the search space remains too large to be comprehensively searched with brute force screening. Our research has, thus, focused on guidance of high throughput experiments via theory-based identification of promising materials systems for photoanode discovery.

The photoanodes identified by our high throughput screening (see SI) include 4 copper vanadates that were identified simultaneously by HiTp theory and experiment. ${ }^{23}$ These initial discoveries motived theory screening of Materials Project entries ${ }^{31}$ based on electronic structure and stability, leading to experimental demonstration of photoanodic activity in 8 additional ternary metal vanadates $^{32}$ and 5 ternary metal manganates. ${ }^{27}$ Experimental screening in composition spaces related to these theory predictions resulted in the identification of an additional 29 photoanode phases. ${ }^{24,33}$ Perhaps the most important lesson from this work is that computational screening not only identifies target phases but also promising composition regions that are sufficiently specific to enable exploration by HiTp experiments, which are in turn sufficiently broad in scope to identify materials beyond the computational search.

The fringe cases in these photoanode discovery campaigns offer insights for guiding future discovery efforts. $\mathrm{FeWO}_{4}$ was identified through combinatorial investigation of non-equilibrium synthesis conditions. ${ }^{26}$ This $\mathrm{Fe}^{+2}$-containing metal oxide is stabilized against oxidation by a selfpassivation surface layer that includes $\mathrm{Fe}^{+3}$, highlighting the role of self-passivation in enabling 
stable operation of thermodynamically unstable photoanodes. Self-passivation was also observed in copper vanadates, where the degradation in photocurrent was found to depend on the thickness of the developed passivating film. ${ }^{34}$ Passive surface films are of intense interest in the metals and alloy industry and provide a fruitful research area in themselves as their formation and functionality are still not entirely understood. ${ }^{35}$ A range of phenomenological models have been developed to explain the evolution of passivation layers, which display varying morphology, shortrange order and chemical constitution depending on the growth process and chemical environment. ${ }^{36}$ The stability of a passivating surface film, and hence that of the underlying bulk material, is explicitly linked to ionic transport through the film which in turn depends critically on the film morphology. Establishing thermodynamic and kinetic criteria for classifying a photoanode as being operationally stable and supporting photoelectrocatalysis is a key area for designing the next-generation of photoanode screening. In previous work, ${ }^{34,} 37$ we have found that wellbenchmarked Pourbaix diagrams ${ }^{38}$ can provide a qualitative guide as to the likely formation of, as well as general chemical composition of, a passive surface film. Furthermore, first-principles methods have been shown to provide quantitative estimates for the relative Gibbs free energy and corresponding aqueous regimes where a candidate photoanode material may form inert passivating films, or steadily corrode to aqueous species. ${ }^{37}$ Detailed understanding of the growth process, evolution, and structure of these complex, self-passivation films is presently lacking, motivating development of new computational and experimental techniques that lead to a predictive model for how the near-surface of a given material will evolve under operational conditions.

The heterogeneous composition and structure of the photoanode as a function of depth from the electrolyte surface complicates the already complex model of semiconductor-liquid junctions, as the effect on these surface layers on band alignment and carrier transport have been insufficiently 
studied to date. The standard thermodynamic requirement of band alignment, i.e. the vacuum energy of the photoanode's valence band being sufficiently negative such that the photo-generated holes are sufficiently oxidizing to drive the OER, is complicated by electrolyte pH-dependent surface dipoles. ${ }^{39}$ The polar surfaces of metal oxides introduce the additional complications of facet and termination-dependent dipoles that alter this band alignment, as exemplified by $\mathrm{BiMn}_{2} \mathrm{O}_{5}{ }^{40}$ where band level calculations for each of 6 low-energy surfaces indicate more than 1.4 $\mathrm{eV}$ variation in work function, making the assessment of band alignment with respect to OER as much of a property of the surface as it is of the bulk electronic structure. Further study of facetdependent and interfacial layer-dependent properties are likely to identify optimal photoanode surfaces and guide synthesis and device implementation by designating desirable and undesirable facets at the electrolyte interface. Initial demonstrations of this concept include facet-dependent charge separation in $\mathrm{BiVO}_{4}{ }^{41}$ and $\mathrm{SrTiO}_{3}{ }^{42}$

This aforementioned $\mathrm{BiMn}_{2} \mathrm{O}_{5},{ }^{40}$ as well as $\beta-\mathrm{Mn}_{2} \mathrm{~V}_{2} \mathrm{O}_{7},{ }^{43}$ provide additional opportunity to investigate performance-limiting attributes of metal oxide photoanodes. The $1.8 \mathrm{eV}$ direct gap of each phase is ideal with respect to solar absorption, but they have yet to be demonstrated as OER photoanodes. While operational stability is a prime suspect for a lack of photoactivity, these are among the most electrochemically stable low-gap metal oxides, with Pourbaix-stable regions in the approximate ranges $0.3-0.7$ and $0.3-1.2 \mathrm{~V}$ vs RHE, respectively. Despite these desirable attributes, these phases are not classified as photoanodes in the present work due to their photoactivity only in the presence of sacrificial hole acceptors. The modest photoactivity obtained with sacrificial hole acceptors also indicates that the photoanode performance limitations extend beyond poor OER catalysis. From an electronic structure point of view, these are exemplary photoanode candidates, motivating detailed inspection as to whether materials optimization can 
confer higher photoactivity, and/or identification of fundamental properties that limit photoactivity with commensurate design of associated screening techniques.

Ternary manganate phases also illustrate the challenges of treating high-temperature or disordered magnetic states in electronic structure calculations. For these ternary manganates, zerotemperature calculations with judiciously-chosen magnetic configurations were required to gauge the possible electronic structure of each phase's ambient temperature paramagnetic state; 40,43 paramagnetic states, in which the local magnetic moments on each open-shell cation are nonzero but their configurational average is zero, are not trivially-compatible with periodic supercells. Typically, high-throughput computational screening employs a computationally less expensive model of the magnetic state, e.g. the ferromagnetic configuration, which can induce significant changes to the electronic structure ${ }^{44}$ of the material compared to the paramagnetic state. In our experience, this approximation can lead to exclusion of promising low-gap metal oxides that exhibit a metallic character in their ferromagnetic state. Antiferromagnetic (AF) ordering is typically a better approximation of the paramagnetic state, and currently the Materials Project is pursuing a large computational survey of the magnetic state of its materials including at least one AF ordering for each transition metal oxide. ${ }^{44}$ Other approaches for computational modelling of paramagnetic materials have been introduced in the literature, ${ }^{45}$ creating an opportunity to evaluate the electronic structure of photoanode materials at relevant operating temperatures.

The compendium of photoanode phases described in the SI offers various opportunities for identifying trends and descriptors for photoactivity. A seldom-discussed materials property that is well characterized by our combinatorial experiments is cation off-stoichiometry of metal oxide photoanodes. Fig. 4 shows the EQE under $3.2 \mathrm{eV}$ illumination for $55 \mathrm{~A}_{1-x} \mathrm{~B}_{x}$ oxide phases, where $\mathrm{B}$ is taken to be the higher valent cation produced by the Materials Project oxidation state 
interpreter, and each phase is plotted by the difference in $x$ between XRF measurements of thin film composition and that of the formula unit (FU). In addition to showing the considerable variation in EQE over the collection of metal oxide photoanodes, the observation of appreciable $\mathrm{EQE}$ at substantial composition deviations is quite striking. There are 5 phases with composition deviation more than 0.18 from the composition of the prototype structure. With this level of composition deviation, nanocrystalline secondary phases (not detected by XRD) may be present, although such composition differences often occur with a multi-valent cation such that the host structure can support substantial alloying. For example, the phases with an excess of the higher valent cation include formal valences $\mathrm{A}^{+2}(\mathrm{~A}=\mathrm{Ca}$ or $\mathrm{Mg})$ and either $\mathrm{Mn}^{+3}$ or $\mathrm{Mn}^{+4}$, where excess Mn appears to alloy as $\mathrm{Mn}^{+2}$ on the $\mathrm{A}^{+2}$ site. There are also cases where the structure of interest is only observed in off-stoichiometric conditions, such as $\mathrm{V}_{2} \mathrm{Ag}_{0.33} \mathrm{O}_{5}$ where an excess of $\mathrm{Ag}$ is needed to form the structure under our synthesis conditions, likely resulting in some metallic Ag in the thin film sample. This level of off-stoichiometry in solar energy conversion materials has been most extensively studied with Cu-based p-type semiconductors such as $\mathrm{Cu}(\mathrm{In}, \mathrm{Ga}) \mathrm{Se}_{2},{ }^{46}$ $\mathrm{Cu}_{2} \mathrm{SnZnS}_{4}{ }^{47}$ and $\mathrm{CuBi}_{2} \mathrm{O}_{4}{ }^{48}$ where alloyed variants improve phase stability with respect to competing phases and/or alter the electronic structure. These phenomena underlie the composition variations of photoanodes in Fig. 4, where alloying can additionally optimize a catalytic activity and/or electrochemical passivation.

The uncertainty in the XRF compositions is nominally 5 at.\%, so phases appearing outside the \pm 0.06 window are confidently off-stoichiometric, bringing into question whether traditional methods would discover these photoanodes. Of the 23 such phases, 7 are also photoactive within the \pm 0.06 window, so discovery may have been possible with synthesis at a composition matching the target FU. The remaining 16 phases required composition deviation to be discovered in our 
experiments, highlighting the utility of composition libraries in photoanode discovery and motivating further study of how substantial alloying optimizes photoanode performance.

Intertwined with the cation off-stoichiometry is the oxygen stoichiometry, or oxygen vacancy concentration, which is not amenable to high throughput characterization, resulting in a lack of observable trends over the set of known photoanodes. Recent work on $\mathrm{BiVO}_{4}$ has demonstrated that over various time scales, the sub-band-gap states created by oxygen vacancies trap holes and electrons, ultimately requiring thermal de-trapping to produce photoactivity. ${ }^{49}$ These results highlight the complexity of carrier transport in metal oxide photoanodes, which often involves small polaron hopping ${ }^{50}$ and may additionally involve more complex phenomena over a range of time scales. An opportunity arising from these recent advances is determination of the extent to which the observed conduction mechanisms of $\mathrm{BiVO}_{4}$ are universal to ternary vanadates and other metal oxide photoanodes, and if the electronic structure contributions of oxygen vacancies, for example, can be used as a functional descriptor of metal oxide photoanodes. Our HiTp screening work includes a concerted exploration of ternary vanadates where we demonstrated tuning of band gap energies across the visible range through band edge hybridization with various open-d-shell cations. ${ }^{23}$ These are specific examples of a general property of metal oxide photoanodes: nontrivial orbital character (particularly d orbital character) at band edges creates opportunities for tuning the band-edge electronic structure in new ways, potentially leading to electron and hole conductivities that are not well described by semi-classical, Boltzmann-based band transport theory.

Traditional semiconductor characterization of conductivity or effective carrier mobility is necessary but insufficient for specific identification of the transport-limiting phenomenon. Recent developments in ultrafast spectroscopy have demonstrated direct observation of polaron formation, ${ }^{51}$ and application of such techniques to a broader class of photoanodes will help 
establish trends in the roles of defects, excited states, etc. in metal oxide semiconductor transport. Disentangling the transport mechanisms can also be facilitated by theory, although the highlylocalized and strongly correlated electronic states (e.g. states with d orbital character) typically require rigorous treatment beyond that of standard theories and computational methods. Metal oxides often exhibit such electronic states, and as noted above, photoanodes of interest often encompass additional structural and chemical complexity that may alter transport properties and may require treatment of many-atom systems, creating substantial computational expense. Although DFT calculations are often used to compute band structures in practice, rigorous calculations of spectroscopic properties of metal oxide photoanode candidates require formalisms beyond the ground-state, time-independent DFT. In materials physics, the formalism of choice for quantitative prediction of the band structure and optical properties is ab initio many-body perturbation theory (MBPT). ${ }^{52} \mathrm{MBPT}$ has been historically computationally prohibitive for complex materials, but it is beginning to be applied to complex systems, such as the metal oxide photoanodes $\mathrm{BiVO}_{4}$ and $\beta-\mathrm{Cu}_{2} \mathrm{~V}_{2} \mathrm{O}_{7},{ }^{22,} 53$ and interfaces involving photoanode materials and water. ${ }^{54}$ Additionally, detailed ab initio calculations of photoexcited carrier dynamics, limited by phonon scattering, are now possible for simple semiconductors, ${ }^{55}$ and have more recently been extended to oxides. ${ }^{56}$ A growing number of recent methodologies are being proposed for more rigorous calculations of polaron formation energies. ${ }^{57}$ Collectively, advances in these methods promise a significantly deeper and richer understanding and assessment of photogenerated carrier phenomena in existing candidate photoanodes, which will also lead to new descriptors for discovery of photoelectrode materials.

Solar fuels photoanodes pose substantial challenges for materials discovery due to the combined needs of solar absorption, charge carrier separation and transport, chemical and electrochemical 
stability under operating conditions, as well as catalytic activity for the OER. The recent increase in chemical diversity of metal oxide photoanodes presents both challenges, for example determining which phases are amenable to optimization and integration into solar fuels generators, and opportunities, for example developing new theory and experiment campaigns to better understand fundamental properties that give rise to photoanodic activity. Despite the prolific photoanode discovery efforts of the last 20 years, solar fuels photoanodes are still rare compared to other types of functional materials, motivating continued identification of such materials to formulate models that relate fundamental materials properties to photoanode performance, enhancing scientific understanding as well as development of deployable solar fuels materials. The photoactivity of off-stoichiometric variants of phases is notable, motivating application of defect and transport characterization techniques, which have been recently developed via study of $\mathrm{Fe}_{2} \mathrm{O}_{3}$ and $\mathrm{BiVO}_{4}$, to a broader set of metal oxide phases. Combining the recent proliferation of both photoanode discoveries and advanced characterization techniques will advance fundamental understanding of metal oxide photoelectrocatalysts and the design of next-generation photoanodes.
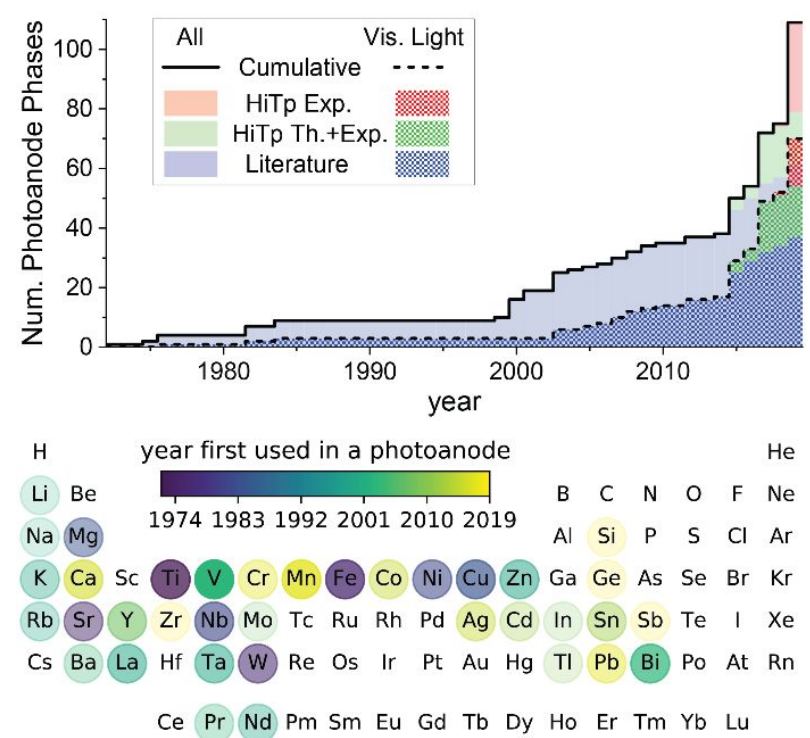

Figure 1: (top) Summary of OER photoanodes from literature (blue), as well as our previous reports integrating HiTp theory and experiment (green) and additional HiTp experiment 


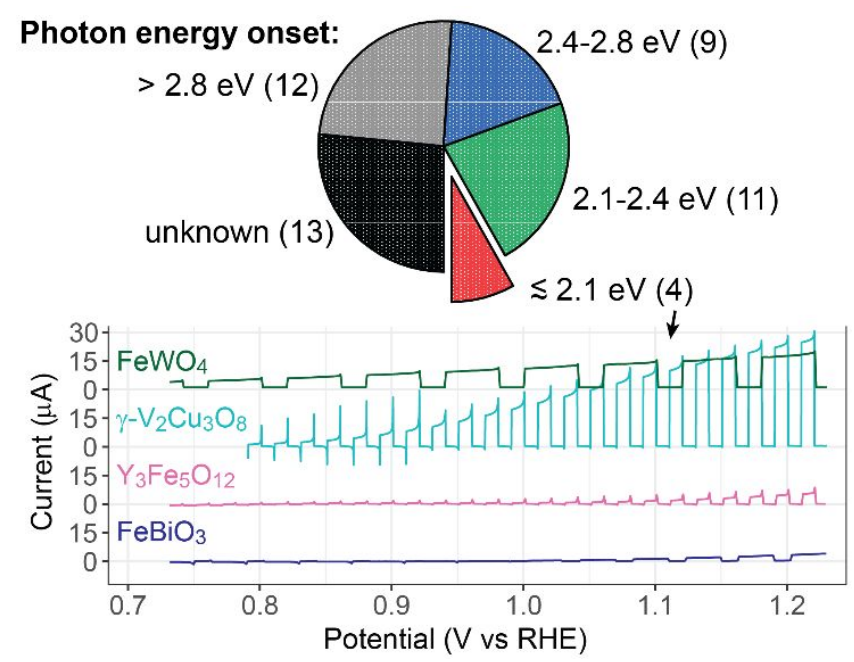

Figure 3: The pie chart shows the distribution of photon energy onset for photoactivity, for 49 metal oxide photoanode phases from combinatorial libraries with EQE in excess of $0.01 \%$ (see SI). The photon energy onset is determined via photoelectrochemistry at $1.23 \mathrm{~V}$ vs RHE with a 
series of light emitting diodes, and due to their spectral breadth the boundaries between the 4 ranges have ca. $\pm 0.1 \mathrm{eV}$ uncertainty. For the 4 photoanodes with photoactivity at $2.1 \mathrm{eV}$, the cathodic sweep from a cyclic voltammogram is shown with toggled $3.2 \mathrm{eV}$ illumination (variable illumination intensity, see SI). These data were acquired in $\mathrm{pH} 13(0.1 \mathrm{M} \mathrm{NaOH})$ for $\gamma-\mathrm{V}_{2} \mathrm{Cu}_{3} \mathrm{O}_{8}$ and $\mathrm{Y}_{3} \mathrm{Fe}_{5} \mathrm{O}_{12}$ and borate-buffered $\mathrm{pH} 9.3$ electrolyte for $\mathrm{FeWO}_{4}$ and $\mathrm{FeBiO}_{3}$, as reported previously. $24,26-27$

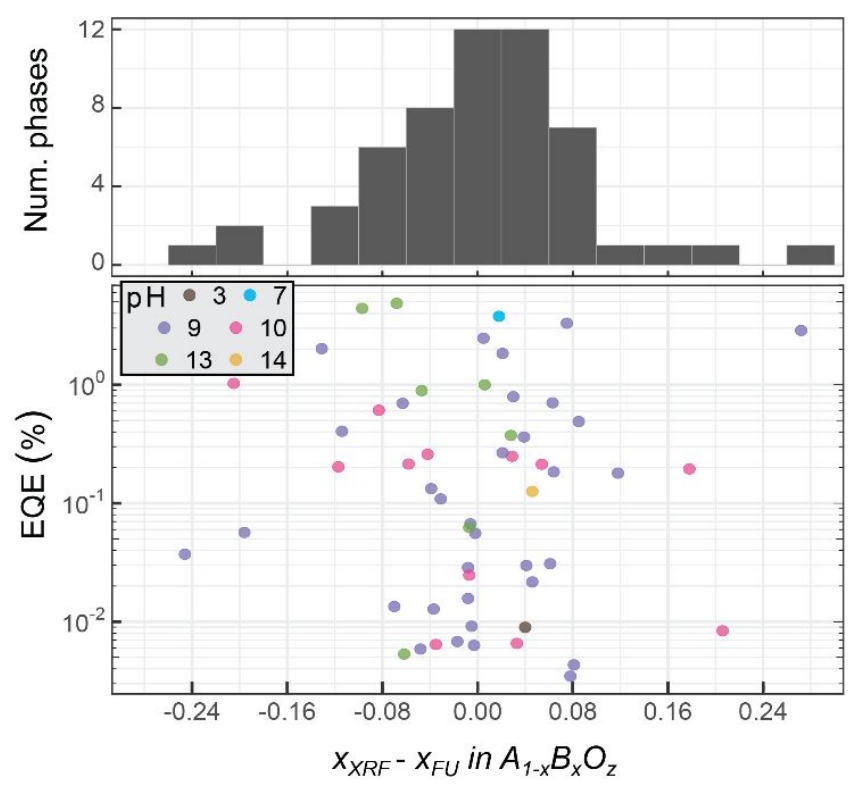

Figure 4: Summary of 55 photoanode phases from combinatorial libraries with available XRF measurement of composition. For each identified ternary oxide, the cation stoichiometry $x$ is taken as $\mathrm{A}_{1-x} \mathrm{~B}_{x} \mathrm{O}_{z}$ where $\mathrm{B}$ is generally the higher valent cation. The quantity $x_{X R F}-x_{F U}$ is the difference in $x$ between the composition of the most photoactive sample and that of the formula unit. The $\mathrm{EQE}$ at $3.2 \mathrm{eV}$ is shown as the metric for photoactivity as this is the only illumination source used for all photoanode samples.

\section{ASSOCIATED CONTENT}


Supporting Information. Table of 109 metal oxide photoanodes from the literature survey summarized in Fig. 1. Table of HiTp composition and photoelectrochemical data for 58 phases measured in our labs.

\title{
AUTHOR INFORMATION \\ *gregoire@caltech.edu
}

\begin{abstract}
Notes
The authors declare no competing financial interest.

$\S$ In the literature survey, photoactivity under white light illumination combined with optical identification of a sub-2.8 eV band gap was considered to be sufficient evidence for a visible light photoanode.
\end{abstract}

\begin{abstract}
AUTHOR BIOGRAPHIES
Lan Zhou earned her Ph.D in Materials Science from University of Vermont in 2010, and is currently working as a Staff Scientist in the High Throughput Experimentation group at Caltech. Her research focuses on developing processes for combinatorial materials synthesis of metals, metal oxides, and mixed anion materials used in solar-fuels applications.
\end{abstract}

Aniketa Shinde specializes in instrumentation, materials science, and electrochemistry for energy research. Her focus is high-throughput scanning drop sensor measurements for photoelectrochemical and electrochemical characterization of thin films. She received her Master's and PhD degrees in Physics from the University of California, Irvine.

Dan Guevarra has been a member of the High Throughput Experimentation group and the Joint Center for Artificial Photosynthesis at Caltech since 2013. He received a Master of Information 
and Data Science from the University of California, Berkeley and presently works on data analysis, visualization, machine learning, and instrument automation.

Joel A. Haber is a Staff Scientist at the California Institute of Technology in the Joint Center for Artificial Photosynthesis. His research focuses on inorganic materials chemistry and highthroughput materials science, as applied to materials and devices for solar-energy conversion.

Kristin A. Persson is an Associate Professor in Materials Science and Engineering at UC Berkeley with a joint appointment as Senior Faculty Scientist at the Lawrence Berkeley National Laboratory. She is also the Director of the Materials Project (www.materialsproject.org) and specializes in materials informatics and data-driven design of novel materials.

Jeffrey B. Neaton is a Professor of Physics at UC Berkeley, and Senior Faculty Scientist and Associate Laboratory Director for Energy Sciences at Lawrence Berkeley National Laboratory. He is also a member of the Kavli Energy Nanosciences Institute at Berkeley. His research focuses on the development and application of electronic structure theory for predictive calculations of properties of inorganic and organic energy and quantum materials.

John Gregoire is the Thrust Coordinator for Photoelectrocatalysis in the Joint Center for Artificial Photosynthesis, a U.S. DOE Energy Innovation Hub. He leads the High Throughput Experimentation group at Caltech, which accelerates scientific discovery by automating critical components of materials research workflows, from synthesis to data interpretation.

ACKNOWLEDGMENT 
This material is based upon work performed by the Joint Center for Artificial Photosynthesis, a

DOE Energy Innovation Hub, supported through the Office of Science of the U.S. Department of

Energy under Award Number DE-SC0004993.

\section{QUOTES TO HIGHLIGHT}

Theoretical guidance of high throughput experiments has been particularly effective in dramatically increasing the portfolio of metal oxide photoanodes, motivating a new era of photoanode development where the characterization and optimization techniques developed on traditional materials are applied to nascent photoanodes that exhibit visible light photoresponse.

Computational screening not only identifies target phases but also promising composition regions that are sufficiently specific to enable exploration by high throughput experiments, which are in turn sufficiently broad in scope to identify materials beyond the computational search.

The photoactivity of off-stoichiometric variants of phases is notable, motivating application of defect and transport characterization techniques, which have been recently developed via study of $\mathrm{Fe}_{2} \mathrm{O}_{3}$ and $\mathrm{BiVO}_{4}$, to a broader set of metal oxide phases.

Combining the recent proliferation of both photoanode discoveries and advanced characterization techniques will advance fundamental understanding of metal oxide photoelectrocatalysts and the design of next-generation photoanodes.

\section{REFERENCES}

1. Kraan, O.; Kramer, G. J.; Haigh, M.; Laurens, C., An Energy Transition That Relies Only on Technology Leads to a Bet on Solar Fuels. Joule 2019, 3 (10), 2286-2290.

2. Raciti, D.; Wang, C., Recent Advances in CO2 Reduction Electrocatalysis on Copper. ACS Energy Letters 2018, 3 (7), 1545-1556.

3. Chen, X.; Li, N.; Kong, Z.; Ong, W.-J.; Zhao, X., Photocatalytic fixation of nitrogen to ammonia: state-of-the-art advancements and future prospects. Materials Horizons 2018, 5 (1), 927.

4. Sayama, K., Production of High-Value-Added Chemicals on Oxide Semiconductor Photoanodes under Visible Light for Solar Chemical-Conversion Processes. ACS Energy Letters 2018, 3 (5), 1093-1101.

5. Mavroides, J. G.; Tchernev, D. I.; Kafalas, J. A.; Kolesar, D. F., Photoelectrolysis of water in cells with TiO2 anodes. Materials Research Bulletin 1975, 10 (10), 1023-1030.

6. Fountaine, K. T.; Lewerenz, H. J.; Atwater, H. A., Efficiency Limits for Photoelectrochemical Water-Splitting. Nat. Commun. 2016, 7, 13706.

7. Tournet, J.; Lee, Y.; Karuturi, S. K.; Tan, H. H.; Jagadish, C., III-V Semiconductor Materials for Solar Hydrogen Production: Status and Prospects. ACS Energy Letters 2020, 611622. 
8. Bae, D.; Seger, B.; Vesborg, P. C. K.; Hansen, O.; Chorkendorff, I., Strategies for stable water splitting via protected photoelectrodes. Chem Soc Rev 2017, 46 (7), 1933-1954.

9. Kim, J. H.; Han, S.; Jo, Y. H.; Bak, Y.; Lee, J. S., A precious metal-free solar water splitting cell with a bifunctional cobalt phosphide electrocatalyst and doubly promoted bismuth vanadate photoanode. Journal of Materials Chemistry A 2018, 6 (3), 1266-1274.

10. Sathre, R.; Greenblatt, J. B.; Walczak, K.; Sharp, I. D.; Stevens, J. C.; Ager, J. W.; Houle, F. A., Opportunities to improve the net energy performance of photoelectrochemical watersplitting technology. Energ Environ Sci 2016, 9 (3), 803-819.

11. Abdi, F. F.; Berglund, S. P., Recent developments in complex metal oxide photoelectrodes. Journal of Physics D: Applied Physics 2017, 50 (19), 193002.

12. Chu, S.; Li, W.; Yan, Y.; Hamann, T.; Shih, I.; Wang, D.; Mi, Z., Roadmap on solar water splitting: current status and future prospects. Nano Futures 2017, 1 (2), 022001.

13. He, H.; Liao, A.; Guo, W.; Luo, W.; Zhou, Y.; Zou, Z., State-of-the-art progress in the use of ternary metal oxides as photoelectrode materials for water splitting and organic synthesis. Nano Today 2019, 100763.

14. (a) Sayama, K.; Nomura, A.; Zou, Z. G.; Abe, R.; Abe, Y.; Arakawa, H., Photoelectrochemical Decomposition of Water on Nanocrystalline $\mathrm{BiVO}_{4}$ Film Electrodes Under Visible Light. Chem. Commun. 2003, (23), 2908-2909; (b) Lamm, B.; Trześniewski, B. J.; Döscher, H.; Smith, W. A.; Stefik, M., Emerging Postsynthetic Improvements of BiVO4 Photoanodes for Solar Water Splitting. ACS Energy Letters 2018, 3 (1), 112-124; (c) Kim, J. H.; Lee, J. S., Elaborately Modified BiVO4 Photoanodes for Solar Water Splitting. Advanced Materials 2019, 31 (20), 1806938.

15. Sharp, I. D.; Cooper, J. K.; Toma, F. M.; Buonsanti, R., Bismuth Vanadate as a Platform for Accelerating Discovery and Development of Complex Transition-Metal Oxide Photoanodes. ACS Energy Letters 2017, 2 (1), 139-150.

16. Zhang, W.; Liu, M., Modulating Carrier Transport via Defect Engineering in Solar Water Splitting Devices. ACS Energy Letters 2019, 4 (4), 834-843.

17. Yang, W.; Prabhakar, R. R.; Tan, J.; Tilley, S. D.; Moon, J., Strategies for enhancing the photocurrent, photovoltage, and stability of photoelectrodes for photoelectrochemical water splitting. Chem Soc Rev 2019, 48 (19), 4979-5015.

18. Jia, X.; Lynch, A.; Huang, Y.; Danielson, M.; Lang'at, I.; Milder, A.; Ruby, A. E.; Wang, H.; Friedler, S. A.; Norquist, A. J.; Schrier, J., Anthropogenic biases in chemical reaction data hinder exploratory inorganic synthesis. Nature 2019, 573 (7773), 251-255.

19. Kölbach, M.; Pereira, I. J.; Harbauer, K.; Plate, P.; Höflich, K.; Berglund, S. P.; Friedrich, D.; van de Krol, R.; Abdi, F. F., Revealing the Performance-Limiting Factors in $\alpha-$ SnWO4 Photoanodes for Solar Water Splitting. Chem Mater 2018, 30 (22), 8322-8331.

20. Abdi, F. F.; Chemseddine, A.; Berglund, S. P.; van de Krol, R., Assessing the Suitability of Iron Tungstate (Fe2WO6) as a Photoelectrode Material for Water Oxidation. The Journal of Physical Chemistry C 2017, 121 (1), 153-160.

21. Jiang, C.-M.; Segev, G.; Hess, L. H.; Liu, G.; Zaborski, G.; Toma, F. M.; Cooper, J. K.; Sharp, I. D., Composition-Dependent Functionality of Copper Vanadate Photoanodes. ACS Applied Materials \& Interfaces 2018, 10 (13), 10627-10633.

22. Wiktor, J.; Reshetnyak, I.; Strach, M.; Scarongella, M.; Buonsanti, R.; Pasquarello, A., Sizable Excitonic Effects Undermining the Photocatalytic Efficiency of $\beta$-Cu2V2O7. The Journal of Physical Chemistry Letters 2018, 9 (19), 5698-5703. 
23. Yan, Q.; Yu, J.; Suram, S. K.; Zhou, L.; Shinde, A.; Newhouse, P. F.; Chen, W.; Li, G.; Persson, K. A.; Gregoire, J. M.; Neaton, J. B., Solar fuels photoanode materials discovery by integrating high-throughput theory and experiment. Proceedings of the National Academy of Sciences of the United States of America 2017, 114 (12), 3040-3043.

24. Zhou, L.; Shinde, A.; Guevarra, D.; Richter, M. H.; Stein, H. S.; Wang, Y.; Newhouse, P.; Persson, K.; Gregoire, J., Combinatorial screening yields discovery of 29 metal oxide photoanodes for solar fuel generation. Journal of Materials Chemistry A 2020, 10.1039/C9TA13829C.

25. Chen, X. Y.; Yu, T.; Gao, F.; Zhang, H. T.; Liu, L. F.; Wang, Y. M.; Li, Z. S.; Zou, Z. G.; Liu, J. M., Application of weak ferromagnetic BiFe O3 films as the photoelectrode material under visible-light irradiation. Appl. Phys. Lett. 2007, 91 (2).

26. Zhou, L.; Shinde, A.; Suram, S. K.; Stein, H. S.; Bauers, S. R.; Zakutayev, A.; DuChene, J. S.; Liu, G.; Peterson, E. A.; Neaton, J. B.; Gregoire, J. M., Bi-Containing n-FeWO4 Thin Films Provide the Largest Photovoltage and Highest Stability for a Sub-2 eV Band Gap Photoanode. ACS Energy Letters 2018, 3 (11), 2769-2774.

27. Zhou, L.; Yan, Q.; Shinde, A.; Guevarra, D.; Newhouse, P. F.; Becerra-Stasiewicz, N.; Chatman, S. M.; Haber, J. A.; Neaton, J. B.; Gregoire, J. M., High Throughput Discovery of Solar Fuels Photoanodes in the CuO-V2O5 System. Adv. Energy Mater. 2015, 5, 1500968. 28. (a) Woodhouse, M.; Herman, G. S.; Parkinson, B. A., Combinatorial Approach to Identification of Catalysts for the Photoelectrolysis of Water. Chem. Mater. 2005, 17 (17), 43184324; (b) Rowley, J. G.; Do, T. D.; Cleary, D. A.; Parkinson, B. A., Combinatorial Discovery Through a Distributed Outreach Program: Investigation of the Photoelectrolysis Activity of pType Fe, Cr, Al Oxides. ACS Applied Materials \& Interfaces 2014, 6 (12), 9046-9052.

29. Jaramillo, T. F.; Baeck, S. H.; Kleiman-Shwarsctein, A.; Choi, K. S.; Stucky, G. D.; McFarland, E. W., Automated electrochemical synthesis and photoelectrochemical characterization of $\mathrm{Zn}_{1-\mathrm{x}} \mathrm{Co}_{\mathrm{x}} \mathrm{O}$ thin films for solar hydrogen production. J. Comb. Chem. 2005, 7 (2), 264-271.

30. (a) Gutkowski, R.; Khare, C.; Conzuelo, F.; Kayran, Y. U.; Ludwig, A.; Schuhmann, W., Unraveling compositional effects on the light-induced oxygen evolution in $\mathrm{Bi}(\mathrm{V}-\mathrm{Mo}-\mathrm{X}) \mathrm{O} 4$ material libraries. Energ Environ Sci 2017, 10 (5), 1213-1221; (b) Meyer, R.; Sliozberg, K.; Khare, C.; Schuhmann, W.; Ludwig, A., High-Throughput Screening of Thin-Film Semiconductor Material Libraries II: Characterization of Fe-W-O Libraries. ChemSusChem 2015, 8 (7), 1279-1285; (c) Lee, J. W.; Ye, H. C.; Pan, S. L.; Bard, A. J., Screening of photocatalysts by scanning electrochemical microscopy. Anal. Chem. 2008, 80 (19), 7445-7450. 31. Jain, A.; Ong, S. P.; Hautier, G.; Chen, W.; Richards, W. D.; Dacek, S.; Cholia, S.; Gunter, D.; Skinner, D.; Ceder, G.; Persson, K. A., The Materials Project: A materials genome approach to accelerating materials innovation. APL Materials 2013, I (1), 011002. 32. Shinde, A.; Suram, S. K.; Yan, Q.; Zhou, L.; Singh, A. K.; Yu, J.; Persson, K. A.; Neaton, J. B.; Gregoire, J. M., Discovery of Manganese-Based Solar Fuel Photoanodes via Integration of Electronic Structure Calculations, Pourbaix Stability Modeling, and HighThroughput Experiments. ACS Energy Letters 2017, 2307-2312.

33. Noh, J.; Kim, S.; Gu, G. h.; Shinde, A.; Zhou, L.; Gregoire, J. M.; Jung, Y., Unveiling new stable manganese based photoanode materials via theoretical high-throughput screening and experiments. Chemical Communications 2019, 55, 13418-13421.

34. Zhou, L.; Yan, Q.; Yu, J.; Jones, R. J. R.; Becerra-Stasiewicz, N.; Suram, S. K.; Shinde, A.; Guevarra, D.; Neaton, J. B.; Persson, K. A.; Gregoire, J. M., Stability and Self-passivation of 
Copper Vanadate Photoanodes under Chemical, Electrochemical, and Photoelectrochemical Operation. Phys. Chem. Chem. Phys. 2016, 18, 9349-9352.

35. (a) Macdonald, D. D., On the Existence of Our Metals-Based Civilization: I. Phase-Space Analysis. Journal of The Electrochemical Society 2006, 153 (7), B213-B224; (b) Macdonald, D. D., Passivity-the key to our metals-based civilization. Pure and Applied Chemistry 1999, 71 (6), 951-978.

36. (a) Macdonald, D. D.; Urquidi-Macdonald, M., Theory of Steady-State Passive Films. Journal of The Electrochemical Society 1990, 137 (8), 2395-2402; (b) Schultze, J. W.; Lohrengel, M. M., Stability, reactivity and breakdown of passive films. Problems of recent and future research. Electrochim Acta 2000, 45 (15), 2499-2513.

37. Singh, A. K.; Zhou, L.; Shinde, A.; Suram, S. K.; Montoya, J. H.; Winston, D.; Gregoire, J. M.; Persson, K. A., Electrochemical Stability of Metastable Materials. Chem Mater 2017, 29 (23), 10159-10167.

38. Persson, K. A.; Waldwick, B.; Lazic, P.; Ceder, G., Prediction of solid-aqueous equilibria: Scheme to combine first-principles calculations of solids with experimental aqueous states. Phys. Rev. B 2012, 85 (23), 235438.

39. Stevanovic, V.; Lany, S.; Ginley, D. S.; Tumas, W.; Zunger, A., Assessing capability of semiconductors to split water using ionization potentials and electron affinities only. Phys Chem Chem Phys 2014, 16 (8), 3706-3714.

40. Newhouse, P. F.; Reyes-Lillo, S. E.; Li, G.; Zhou, L.; Shinde, A.; Guevarra, D.; Suram, S. K.; Soedarmadji, E.; Richter, M. H.; Qu, X.; Persson, K.; Neaton, J. B.; Gregoire, J. M., Discovery and Characterization of a Pourbaix-Stable, 1.8 eV Direct Gap Bismuth Manganate Photoanode. Chem Mater 2017, 29 (23), 10027-10036.

41. Li, R.; Han, H.; Zhang, F.; Wang, D.; Li, C., Highly efficient photocatalysts constructed by rational assembly of dual-cocatalysts separately on different facets of BiVO4. Energ Environ Sci 2014, 7 (4), 1369-1376.

42. Mu, L.; Zhao, Y.; Li, A.; Wang, S.; Wang, Z.; Yang, J.; Wang, Y.; Liu, T.; Chen, R.; Zhu, J.; Fan, F.; Li, R.; Li, C., Enhancing charge separation on high symmetry SrTiO3 exposed with anisotropic facets for photocatalytic water splitting. Energ Environ Sci 2016, 9 (7), 2463 2469.

43. Yan, Q.; Li, G.; Newhouse, P. F.; Yu, J.; Persson, K. A.; Gregoire, J. M.; Neaton, J. B., Mn2V2O7: An Earth Abundant Light Absorber for Solar Water Splitting. Advanced Energy Materials 2015, 5 (8), 1401840.

44. Horton, M. K.; Montoya, J. H.; Liu, M.; Persson, K. A., High-throughput prediction of the ground-state collinear magnetic order of inorganic materials using Density Functional Theory. npj Computational Materials 2019, 5 (1), 64.

45. (a) Mozafari, E.; Alling, B.; Steneteg, P.; Abrikosov, I. A., Role of N defects in paramagnetic $\mathrm{CrN}$ at finite temperatures from first principles. Physical Review B 2015, 91 (9), 094101; (b) Abrikosov, I. A.; Ponomareva, A. V.; Steneteg, P.; Barannikova, S. A.; Alling, B., Recent progress in simulations of the paramagnetic state of magnetic materials. Current Opinion in Solid State and Materials Science 2016, 20 (2), 85-106; (c) Trimarchi, G.; Wang, Z.; Zunger, A., Polymorphous band structure model of gapping in the antiferromagnetic and paramagnetic phases of the Mott insulators $\mathrm{MnO}, \mathrm{FeO}, \mathrm{CoO}$, and NiO. Physical Review B 2018, 97 (3), 035107.

46. Ramanujam, J.; Singh, U. P., Copper indium gallium selenide based solar cells - a review. Energy \& Environmental Science 2017, 10 (6), 1306-1319. 
47. Dhawale, D. S.; Ali, A.; Lokhande, A. C., Impact of various dopant elements on the properties of kesterite compounds for solar cell applications: a status review. Sustainable Energy \& Fuels 2019, 3 (6), 1365-1383.

48. Sharma, G.; Zhao, Z.; Sarker, P.; Nail, B. A.; Wang, J.; Huda, M. N.; Osterloh, F. E., Electronic structure, photovoltage, and photocatalytic hydrogen evolution with p-CuBi2O4 nanocrystals. Journal of Materials Chemistry A 2016, 4 (8), 2936-2942.

49. Selim, S.; Pastor, E.; García-Tecedor, M.; Morris, M. R.; Francàs, L.; Sachs, M.; Moss, B.; Corby, S.; Mesa, C. A.; Gimenez, S.; Kafizas, A.; Bakulin, A. A.; Durrant, J. R., Impact of Oxygen Vacancy Occupancy on Charge Carrier Dynamics in BiVO4 Photoanodes. Journal of the American Chemical Society 2019, 141 (47), 18791-18798.

50. Rettie, A. J. E.; Chemelewski, W. D.; Emin, D.; Mullins, C. B., Unravelling SmallPolaron Transport in Metal Oxide Photoelectrodes. The Journal of Physical Chemistry Letters 2016, 7 (3), 471-479.

51. Carneiro, L. M.; Cushing, S. K.; Liu, C.; Su, Y.; Yang, P.; Alivisatos, A. P.; Leone, S. R., Excitation-wavelength-dependent small polaron trapping of photoexcited carriers in $\alpha-\mathrm{Fe} 2 \mathrm{O} 3$. Nature Materials 2017, 16 (8), 819-825.

52. (a) Strinati, G., Application of the Green's functions method to the study of the optical properties of semiconductors. La Rivista del Nuovo Cimento (1978-1999) 1988, 11 (12), 1-86; (b) Onida, G.; Reining, L.; Rubio, A., Electronic excitations: density-functional versus manybody Green's-function approaches. Reviews of Modern Physics 2002, 74 (2), 601-659; (c) Golze, D.; Dvorak, M.; Rinke, P., The GW Compendium: A Practical Guide to Theoretical Photoemission Spectroscopy. Frontiers in Chemistry 2019, 7, 377.

53. Wiktor, J.; Reshetnyak, I.; Ambrosio, F.; Pasquarello, A., Comprehensive modeling of the band gap and absorption spectrum of BiVO4. Physical Review Materials 2017, 1 (2), 022401.

54. (a) Ambrosio, F.; Wiktor, J.; Pasquarello, A., pH-Dependent Catalytic Reaction Pathway for Water Splitting at the BiVO4-Water Interface from the Band Alignment. ACS Energy Letters 2018, 3 (4), 829-834; (b) Gerosa, M.; Gygi, F.; Govoni, M.; Galli, G., The role of defects and excess surface charges at finite temperature for optimizing oxide photoabsorbers. Nature Materials 2018, 17 (12), 1122-1127.

55. (a) Bernardi, M.; Vigil-Fowler, D.; Lischner, J.; Neaton, J. B.; Louie, S. G., Ab Initio Study of Hot Carriers in the First Picosecond after Sunlight Absorption in Silicon. Physical Review Letters 2014, 112 (25), 257402; (b) Bernardi, M.; Vigil-Fowler, D.; Ong, C. S.; Neaton, J. B.; Louie, S. G., Ab initio study of hot electrons in GaAs. Proceedings of the National Academy of Sciences 2015, 112 (17), 5291-5296.

56. (a) Zhou, J.-J.; Hellman, O.; Bernardi, M., Electron-Phonon Scattering in the Presence of Soft Modes and Electron Mobility in SrTiO3 Perovskite from First Principles. Physical Review Letters 2018, 121 (22), 226603; (b) Zhou, J.-J.; Bernardi, M., Predicting charge transport in the presence of polarons: The beyond-quasiparticle regime in SrTiO3. Physical Review Research 2019, 1 (3), 033138 .

57. Sio, W. H.; Verdi, C.; Poncé, S.; Giustino, F., Polarons from First Principles, without Supercells. Physical Review Letters 2019, 122 (24), 246403. 